\title{
EchoGéo
}

36 | 2016

Stratégies de villes et "modèles urbains"

\section{La géographie au-delà des frontières disciplinaires}

Jean-Louis Chaléard

\section{(2) OpenEdition}

Journals

Electronic version

URL: https://journals.openedition.org/echogeo/14641

DOI: 10.4000/echogeo.14641

ISSN: 1963-1197

\section{Publisher}

Pôle de recherche pour l'organisation et la diffusion de l'information géographique (CNRS UMR 8586)

\section{Electronic reference}

Jean-Louis Chaléard, "La géographie au-delà des frontières disciplinaires", EchoGéo [Online], 36 | 2016, Online since 30 June 2016, connection on 10 August 2021. URL: http://journals.openedition.org/ echogeo/14641 ; DOI: https://doi.org/10.4000/echogeo.14641

This text was automatically generated on 10 August 2021

EchoGéo est mis à disposition selon les termes de la licence Creative Commons Attribution - Pas d'Utilisation Commerciale - Pas de Modification 4.0 International (CC BY-NC-ND) 


\title{
La géographie au-delà des frontières disciplinaires
}

\author{
Jean-Louis Chaléard
}

1 La nouvelle livraison d'Échogéo est nourrie de deux dossiers relativement volumineux, l'un sur les modèles urbains et l'autre sur la géoarchéologie. Au-delà de leurs grandes différences, dans leurs champs comme leurs objectifs, chacun pose à sa façon la question des rapports entre la géographie et d'autres disciplines. Le premier aborde des thèmes, entre autres sur les stratégies des villes et les politiques ou l'aménagement urbains, qui ont fait l'objet de nombreuses recherches de non-géographes : historiens, politistes, architectes, urbanistes, etc. Le second analyse l'émergence d'une nouvelle discipline scientifique, à l'articulation de la géographie et de l'archéologie. Tous deux permettent d'inscrire la démarche géographique au cœur des investigations les plus récentes en sciences humaines et sociales.

2 Le dossier Sur le champ proposé par É. Peyroux et T. Sanjuan porte sur «Stratégies de villes et «modèles » urbains : approche économique et géopolitique des relations entre villes ». La longue introduction permet de replacer les différentes contributions dans le cadre du thème abordé et dans leur contexte scientifique. Il s'agit de voir, notamment, comment la production et la circulation de modèles urbains participent des stratégies internationales des villes et contribuent au développement de nouvelles relations entre elles. Il s'agit de voir aussi en quoi et comment la circulation des modèles urbains renouvelle les catégories et les fonctions des villes, et tisse des liens inédits entre elles. Le sujet est abordé dans sa grande diversité, comme en témoignent les nombreux « modèles » mobilisés (« ville créatrice », « smart cities », « urbanisme tactique », etc.). La variété des cas retenus permet également de ne pas se limiter à l'examen des grandes métropoles, mais de voir comment les modèles s'appliquent aussi dans les villes moyennes (Saint-Étienne), voire des petites villes (évoquées parmi les villes nordiques). L'étude de deux métropoles du Sud (Johannesburg et Amman) invite à s'interroger sur l'adaptation de modèles importés du Nord, mais aussi sur les innovations dont sont capables de faire preuve ces villes. 
3 Un autre intérêt du dossier est d'associer un questionnement sur l'économie avec un autre sur la géopolitique, dans différentes dimensions, que ce soit l'interrogation sur les politiques néolibérales ou sur l'émergence de nouveaux modèles en rupture avec ces politiques. Le niveau le plus souvent analysé est celui de l'international, avec des stratégies de métropoles qui essaient de valoriser leur image à l'extérieur, dans un contexte de compétition entre grandes cités, et/ou avec une volonté d'orienter le développement urbain mondial (cas de Johannesburg et des villes nordiques par exemple). Mais d'autres échelles sont aussi abordées: les niveaux macrorégional ou national (avec, entre autres, le cas d'Amman qui essaie de s'émanciper de la tutelle d'un État centralisateur et de s'affirmer dans sa région), voire le niveau local (pris en compte dans «l'urbanisme tactique»), les différentes échelles étant souvent liées (comme le montre bien, aussi, le cas des « smart cities»).

列 en présentant les principaux modèles urbains qui se sont succédé depuis la "ville fonctionnelle» de Le Corbusier. Les deux articles suivants se penchent sur la «ville durable», avec l'exemple des villes nordiques qui instrumentalisent à l'échelle mondiale un modèle fondé sur la protection et la valorisation de l'environnement (C. Girault) et l'exemple d'Amman, cité du Sud, qui met en place une nouvelle politique de transports urbains (E. Ducharme). G. Sechi, à partir de l'examen de la «ville créative » dans le cas de Saint-Étienne, et R. Languillon-Aussel, N. Leprêtre, B. Garnier à partir du cas des "smart cities» dans le contexte japonais, s'interrogent sur l'apparition de nouvelles formes de modèles. Le texte d'É. Peyroux montre, à travers la stratégie de relations internationales de Johannesburg, que le contenu des politiques et la sélection des villes partenaires sont en partie conditionnés par les objectifs géopolitiques du gouvernement sud-africain. La démarche de N. Douay et M. Prévot, qui termine le dossier apporte un contrepoint et un complément aux autres cas étudiés en abordant le modèle urbain par le bas, à partir de l'analyse de "l'urbanise tactique » qui doit permettre aux citadins de prendre en charge et d'améliorer leur environnement immédiat.

5 La rubrique Sur le métier propose dans ce numéro d'Échogéo un dossier sur la géoarchéologie avec plusieurs contributions. Dans leur introduction, J. Burnouf et L. Gillot offrent une réflexion sur l'interdisciplinarité, et s'interrogent sur les champs, les concepts et outils partagés par les géographes et les archéologues. Elles mettent en évidence les liens entre géographie et archéologie, et comment est née la géoarchéologie. Le dossier est structuré en deux parties. La première est composée de deux textes rédigés par les responsables du dossier. J. Burnouf, archéologue médiéviste, dissèque les relations entre les métiers de géographes et d'archéologues. L. Gillot, qui a été initiée à différentes disciplines dans sa formation (histoire, archéologie, géographie), s'interroge sur l'articulation entre le savoir scientifique et le savoir endogène à partir de ses recherches de terrain dans l'oasis de Figuig au Maroc. La deuxième partie du dossier est un entretien réalisé avec deux chercheuses, H. Noizot et S. Robert, qui évoquent, à travers leur parcours scientifique ce qui lie géographie et archéologie et ce que la géographie apporte à leurs pratiques d'archéologues.

6 Nous avions déjà évoqué la mémoire de notre collègue Matthieu Giroud, victime des attentats du 13 novembre 2015. Suite à la journée qui lui a été consacrée le 17 mai 2016 à l'Université Paris Est Marne-la-Vallée, M. Delage et S. Weber lui rendent hommage ici, dans la rubrique Sur l'écrit, à partir de l'analyse de ses travaux et de son apport 
scientifique. Les auteurs insistent sur l'importance de l'écriture collective dans son œuvre. Ils soulignent aussi son travail d'édition critique de travaux hors du champ francophone et son activité de traducteur. Matthieu Giroud travaillait sur le changement urbain, attentif aux théories urbaines, mais plus encore soucieux de l'allerretour indispensable avec le travail empirique de terrain. Cela l'amenait autant à se fondre dans la ville avec ses habitants qu'à mener une réflexion critique sur les modes de développement urbains. Par sa pratique collective et fondée sur le contact avec le terrain, par le contenu ouvert de ses recherches sur la ville, il aurait sans doute été en phase avec le présent numéro d'Échogéo. 